\title{
A PILOT STUDY OF THE INTRACRANIAL MULTILAYER FLOW MODULATOR AS A NEW DISRUPTIVE TECHNOLOGY IN THE TREATMENT OF CEREBRAL ANEURYSMS
}

\author{
[SHORT TITLE] \\ INTRACRANIAL MULTILAYER FLOW MODULATOR
}

Sherif Sultan, MCh, MD, FRCS, EBQS/Vasc, $\mathrm{PhD}^{1,2}$; Edel P Kavanagh, BSc, $\mathrm{PhD}^{2}$; Antoine Alves $\mathrm{MD}^{3}$; Isabel Wanke, $\mathrm{MD}^{4}$; Sophia Goericke $\mathrm{MD}^{4}$; Daniel Ruefenacht MD ${ }^{5}$; Niamh Hynes, MD, MRSC ${ }^{2}$

${ }^{1}$ Western Vascular Institute, Department of Vascular and Endovascular Surgery, University Hospital Galway, Newcastle Road, Galway, Ireland.

${ }^{2}$ Department of Vascular Surgery, Doughiska, Galway, Ireland.

${ }^{3}$ NAMSA, Chasse-sur-Rhone, France.

${ }^{4}$ University Hospital Essen, Institute of Diagnostic and Interventional Radiology and Neuroradiology, Hufelandstrasse 55, 45147 Essen, Germany.

${ }^{5}$ Neuroradiology, Swiss Neuro Institute, Clinic Hirslanden, Witellikerstrasse 40, CH-8032 Zurich, Switzerland.

Corresponding author: Sherif Sultan, MCh, MD, Suite 24, Department of Vascular Surgery, Galway Clinic, Doughiska, Galway.

Telephone: +35391720122 . Fax: +35391785871 .

E-mail: sherif.sultan@hse.ie

\section{Conflict of interest}

Isabel Wanke, Sophia Goericke and Daniel Ruefenacht received funding from Cardiatis (Isnes, Belgium) to carry out the animal study.

Antoine Alves received funding from Cardiatis (Isnes, Belgium) to carry out the specimen analysis 


\section{ABSTRACT}

The purpose of this study was to evaluate by histopathological analysis and Energy Dispersive X-ray analysis, the ability to occlude cerebral aneurysms with the Cardiatis (Isnes, Belgium) intracranial Multilayer Flow Modulator (MFM) device.

The devices were implanted in the subclavian artery distally to the brachiocephalic trunk proximally of 6 rabbit animal models, following induction of an aneurysm in the right carotid artery, collateral to the implanted segment of the subclavian. Device placement was performed 4 weeks after aneurysm creation, and for an implantation duration of 4 weeks. The aim of the study was to demonstrate local biocompatibility of the device as well as device integration without signs of complication.

Gross examination and histopathological analysis showed the aneurysmal areas of all six specimens were obturated with an old mixed thrombus, showing the lines of Zahn. The devices were also markedly integrated into the arterial wall with no evidence of local intolerance, and no aneurysm rupture or leakage was observed. Energy dispersive x-ray analysis of the specimens revealed that in all areas investigated in the tissues surrounding the device struts, there was an absence of metal release.

These results demonstrate the basic mechanism of the MFM, its ability to occlude a cerebral aneurysm, created using a rabbit model. Studies using a larger cohort and longer time period are necessary to assess the intracranial MFM's long-term results.

Key words: Intracranial aneurysm; aneurysm; Animal models; Prostheses and Implants 


\section{INTRODUCTION}

The purpose of this study was to evaluate by histopathological analysis, the ability to occlude cerebral aneurysms with the Cardiatis (Isnes, Belgium) intracranial Multilayer Flow Modulator (MFM) device, without causing substantial parent or small branch artery compromise. The MFM is a self-expandable mesh of cobalt alloy wires interconnected in five layers (Figure 1). The MFM is extremely flexible with a low total porosity. It is hypothesized that its design allows blood flow through the device mesh to maintain collateral branch patency, while modulating turbulent flow to laminar within the device and the aneurysm sac. The laminar flow in the aneurysm reduces shear stress on the aneurysm wall and encourages an organized thrombus formation, thereby stabilizing aneurysm size and reducing risk of rupture (Sultan et al. 2012)

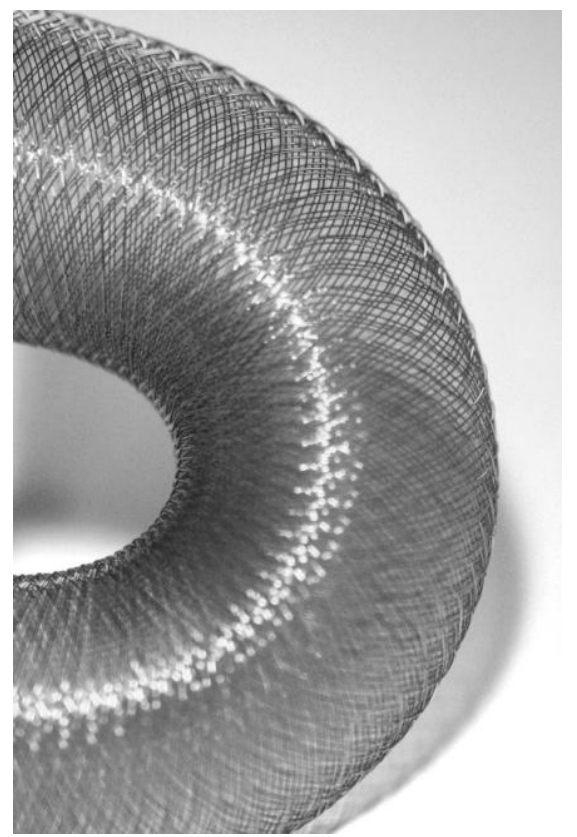

Figure 1 The Multilayer flow modulator device (Cardiatis, Isnes, Belgium)

Intracranial devices are widely used in the treatment of intracranial aneurysms. The study device is similar in concept to intracranial flow diverter devices, which are currently available for clinical use (Lubicz et al. 2010; Piotin et al. 2010; Byrne et al. 2012). Self- expandable intracranial stents are increasingly used to treat complex aneurysms such as widenecked and fusiform aneurysms (Lubicz et al. 2010; Piotin et al. 2010; Byrne et al. 2012).

Flow diverters available include the Pipeline Embolization Device (ev3, Plymouth, MN, US), a self-expanding, microcatheter-delivered device; the SILK Arterial Reconstruction Device (Balt Extrusion, Montmorency, France), to be used with embolization coils. According to Wong et al. (2011), the ultimate treatment goal for intracranial aneurysms is to reconstruct the vessel wall and correct the hemodynamic disturbance. A flow diverter has the ability to do this. The ultimate question is can flow diverters replace coil embolization for the treatment of all aneurysms, such as wide-necked, fusiform, large, and giant unruptured, or selected intracranial aneurysms (Wong et al. 2011).

The MFM has previously received 
CE marking for use in peripheral and aortic aneurysm repair. Many of these devices have been placed in patients on compassionate grounds under strict IFU, however little is known in relation to the mechanisms of action of the device.

\section{METHODS}

The animal test application was made as follows: Application No. 50.05230-48/06 - G883/06, entitled "Validation of new endovascular methods and materials for prevention and treatment of bleeding in the brain within the aneurysm therapy (endovascular aneurysm therapy)". Permission was sought and subsequently approved by the State Agency for Nature, Environment and Consumer Protection of the North Rhine-Westphalia State Environment Agency (Germany). Six rabbit models of type Chinchilla Bastard Rabbit (Charles River Laboratories, Sulzfeld, Germany) were used for this study. Inclusion criteria for the animals required that they were of the same type, weight $(3-4.5 \mathrm{~kg})$ and in good health. Because this was not a comparative study, the animal cohort alphabetical letter assignments (Animals A-F) were not randomized or blinded during the surgical procedure.

The animals first received a preanesthetic of ketamine $10 \%(3.5 \mathrm{ml})$ and xylazine $2 \%(1.5 \mathrm{ml})$, followed by anesthesia with propofol $1 \%(3-4 \mathrm{ml} / \mathrm{kg}$ per hour) and a bolus of pre-anesthesia agent. The aneurysm was created in each of the rabbit models by ligation of the right carotid artery, followed by elastase injection. The aneurysm was then allowed to develop over a period of 4 weeks prior to device implantation. The devices were subsequently implanted in the subclavian artery devices were implanted in the subclavian artery distally to the brachiocephalic trunk proximally of 6 rabbit models, collateral to the implanted segment of the subclavian. Device implantation was performed 4 weeks after aneurysm creation, for a duration of 4 weeks. The rabbit appears to be the most appropriate animal model for the size and geometry of the created aneurysm is similar to those encountered in the human brain (Bouzeghrane et al. 2010). During preoperative treatment, the animals were treated for 2 days with aspirin before implantation (0.5 $\mathrm{ml}$ per day). Upon implantation, heparin (300 UI / kg) was administered. After implantation the animals also received antiplatelet therapy for 30 days $(0.5 \mathrm{ml}$ intravenous aspirin, 100 units of heparin). The animals were euthanized with pentobarbital $(5 \mathrm{ml})$ on the day of completion of the study.

\subsection{Device Explantation}

Rabbit A, B, C, D, E and F had 1 device implanted at the level of the created aneurysm. Upon sacrifice of the animals at 4 weeks post implantation, the arteries and devices were explanted for histological and EDAX analysis. The specimens were stored, immersed in $10 \%$ buffered formalin solution at room temperature $\left(+15^{\circ} \mathrm{C} /+\right.$ $\left.25^{\circ} \mathrm{C}\right)$.

\subsection{Gross Examination}

A gross examination of the external and internal surface of each of the 6 specimens was performed. Device incorporation (in device covering tissues) and signs of local intolerance such as thrombus, inflammation, hemorrhage, intra-device stenosis and patency were evaluated, if present. 


\subsection{EDAX analysis}

A portion of the specimen explanted from rabbit $\mathrm{C}$ was post-fixed in buffered glutaraldehyde solution and dehydrated in $20 \%$ acetone solution. It was then submitted to the critical point process and metallized with gold palladium covering. EDAX was performed using a Hitachi 8800 microscope (Hitachi Corporation, Tokyo, Japan), under $15 \mathrm{KeV}$ voltage. Release of the device materials, nickel and titanium, components of the cobalt alloy were examined in the tissues surrounding the device struts. The noncovered area of the device was also investigated to obtain control values.

\subsection{Histological Preparation and Examination}

The specimens explanted from all 6 animals were dehydrated in alcohol baths of increasing concentrations, washed in xylene and embedded in polymethylmethacrylate resin. The histological sections were obtained using a microcutting and grinding technique (Donath et al. 1982). One central longitudinal section was prepared per specimen. The sections were then stained (Paragon staining) for histopathological examination. The histopathological analysis included the following parameters: inflammatory cells, necrosis, fibrin and thrombus deposits, endothelialization, neointimal hyperplasia and cellularity, smooth muscle cells or fibroblast growth, neovessels, integration of the device, elastic lamina rupture, protrusion, medial alteration and obturation of the aneurysmal area. Parameters for each animal were recorded on a 0 to 4 scale of severity. Specific statistical methods were not used in this study due to the small sample size. The analysis was performed using a Nikon microscope Eclipse E600 coupled with a Nikon DN100 digital camera (Nikon Corporation, Tokyo, Japan).

\section{RESULTS}

For each deployed device, no major difficulty was encountered during placement. The devices were correctly delivered and deployed to the target sites. No animal was excluded from the study and all results are reported.

\subsection{Gross Examination}

No abnormality was observed externally with the 6 specimens. All specimens showed some red thrombus deposit ranging from a moderate to a marked grade of deposition in the subclavian segment. A red thrombus deposit was evident in the subclavian region of rabbit A. A thick parietal red thrombus was evident in rabbit B. A full obturation of the subclavian segment with red thrombus was evident in rabbit C, D, E and $\mathrm{F}$ (Figure 2). Due to the red thrombus deposits, it was not possible to evaluate intra-device covering tissues, signs of inflammation, hemorrhage and intra-device stenosis. 


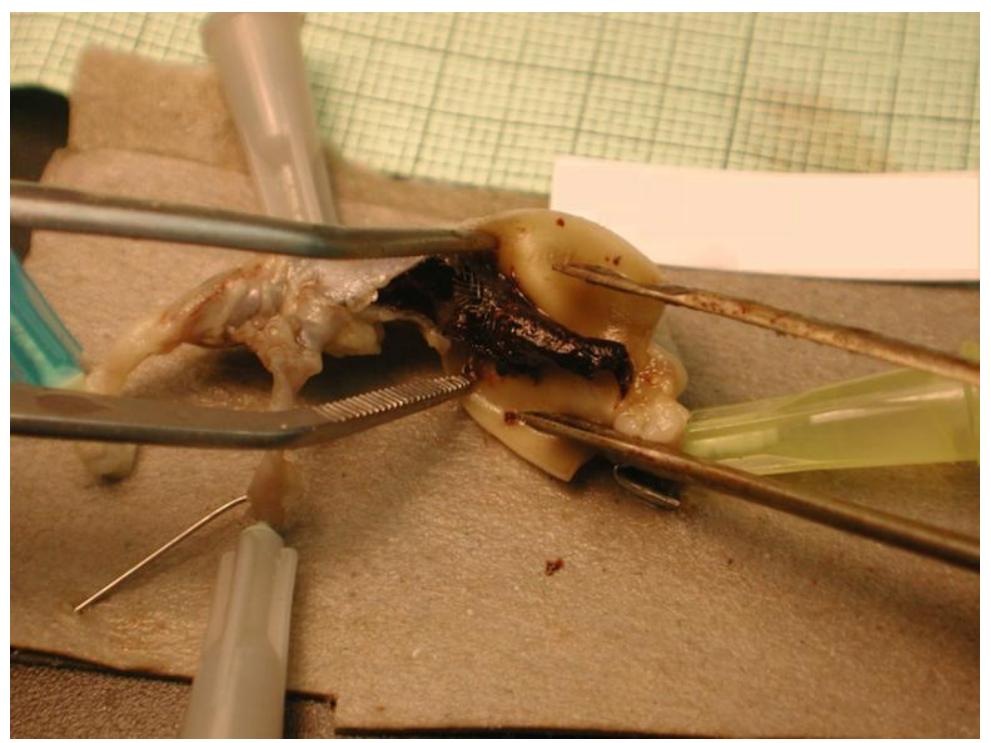

Figure 2 Gross examination of rabbit B showing obturation of the subclavian segment with a red thrombus.

\subsection{Histological Analysis}

The semi-quantitative analysis is presented in Table 1. The thrombi observed macroscopically in all specimens were interpreted as agonal thrombus. The specimen from rabbit A showed that lysis of the elastic lamina for induction of the aneurysm was not effective as the elastic lamina layers were fully preserved. There were marked signs of integration of the device into the vascular wall. The aneurysmal area was fully obturated by a mixed thrombus showing the lines of Zahn, which are associated with thrombus remodeling (Figure 3A). There was a moderate grade of necrosis, and signs of degeneration within the aneurysmal sac. There was also a slight inflammatory cell response including macrophages, giant cells and neovessel infiltration, with a slight neointimal thickening and cellularity around the device. A slight medial thinning was also observed. The specimen from rabbit $\mathrm{B}$ showed that lysis of the elastic lamina for induction of aneurysm was effective as the elastic lamina layers were fully degenerated in the right carotid. The device was slightly integrated in the vascular tissue. The aneurysmal area was also fully obturated by a mixed thrombus showing the lines of Zahn (Figure 3B). There was a moderate grade of necrosis and signs of degeneration within the aneurysmal sac. There was also a slight to moderate inflammatory cell response including macrophages, giant cells and neovessel infiltration, with a slight neointimal thickening and cellularity. A slight medial thinning was also observed.

TABLE 1. Semi-Quantitative Histopathological Analysis*

\begin{tabular}{lllllllc}
\hline Rabbit & A & B & C & D & E & F & Mean \\
\hline Elastic lamina lysis (induction) & 0 & 3 & 2 & 0 & 0 & 2 & 1.2 \\
Mural thrombus & 0 & 0 & 0 & 0 & 0 & 0 & 0 \\
Fibrin deposit & 4 & 4 & 3 & 3 & 3 & 3 & 3.3 \\
Necrosis (aneurysm) & 2 & 2 & 2 & 0 & 0 & 0 & 1 \\
Degeneration (aneurysm) & 2 & 2 & 2 & 0 & 0 & 0 & 1
\end{tabular}




\begin{tabular}{llllllll} 
Polymorphonuclear cells & 0 & 0 & 0 & 0 & 0 & 0 & 0 \\
Lymphocytes & 0 & 0 & 0 & 0 & 0 & 0 & 0 \\
Plasma cells & 0 & 0 & 0 & 0 & 0 & 0 & 0 \\
Macrophages & 1 & 2 & 2 & 1 & 1 & 1 & 1.3 \\
Giant cells & 1 & 1 & 1 & 1 & 1 & 1 & 1 \\
Neovessels & 1 & 2 & 2 & 2 & 2 & 2 & 1.8 \\
Neointimal thickness & 1 & 1 & 1 & 1 & 1 & 1 & 1 \\
Neointimal cellularity & 1 & 1 & 1 & 1 & 1 & 1 & 1 \\
Fibrocytes or smooth muscle cells & 0 & 1 & 1 & 1 & 1 & 1 & 0.8 \\
Device integration & 3 & 3 & 2 & 1 & 1 & 1 & 1.8 \\
Protrusion & 0 & 0 & 0 & 0 & 0 & 0 & 0 \\
Medial thinning & 1 & 1 & 1 & 1 & 1 & 1 & 1 \\
Material degradation & 0 & 0 & 0 & 0 & 0 & 0 & 0 \\
\hline
\end{tabular}

*Mean of ratings on a 5-point scale: $0=$ absence; $1=$ slight; $2=$ moderate; 3 = marked; $4=$ severe/full.

Rabbit C showed partial signs of lysis of the elastic lamina. The device was slightly integrated into the vascular wall. The aneurysmal area was fully obturated by a mixed thrombus showing the lines of Zahn that were associated with signs of peripheral thrombus remodeling (Figure 3C). No signs of leakage were observed. In rabbit $\mathrm{D}$ and $\mathrm{E}$, a tangential section of the aneurysmal branch could be observed. The lysis of the elastic lamina was not effective but the carotid was obturated by a remodeled thrombus, showing characteristics of a vascularized fibroconnective tissue associated with a few hemosiderin residues (Figure 4A, Figure 4B). All other side branches were patent. The device was partially integrated into the vascular wall. Where the device was not integrated, a neointimal tissue growth was observed underneath the device frame. No signs of leakage were observed. In rabbit F, similar observations as per rabbit $\mathrm{E}$ were noted (remodeled thrombus - Figure 4C) with the exception of a few signs of elastolysis observed in the carotid wall.

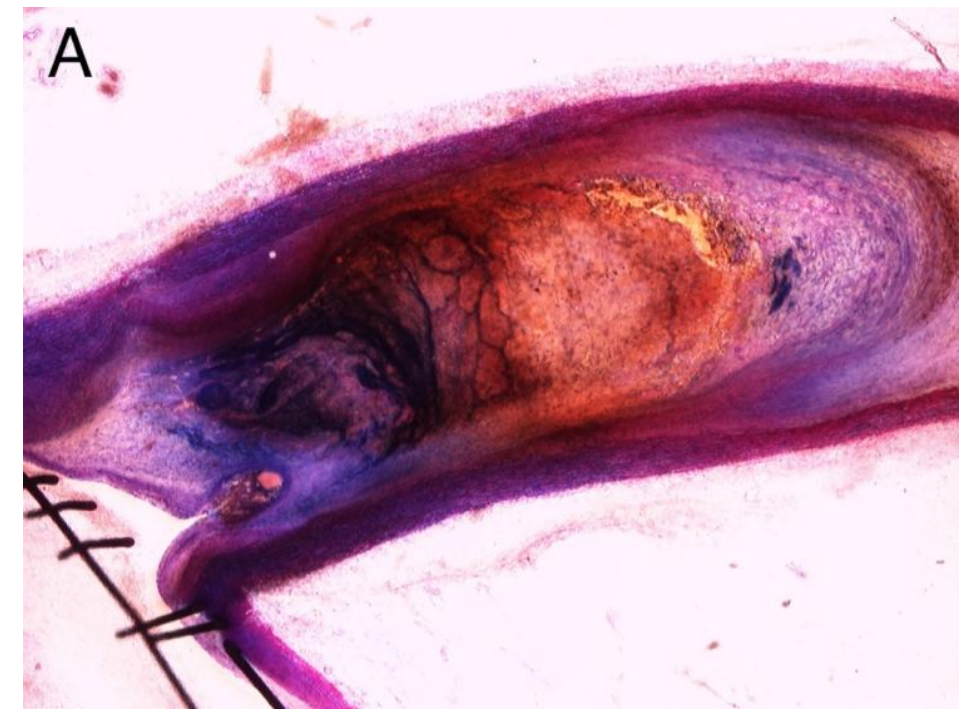



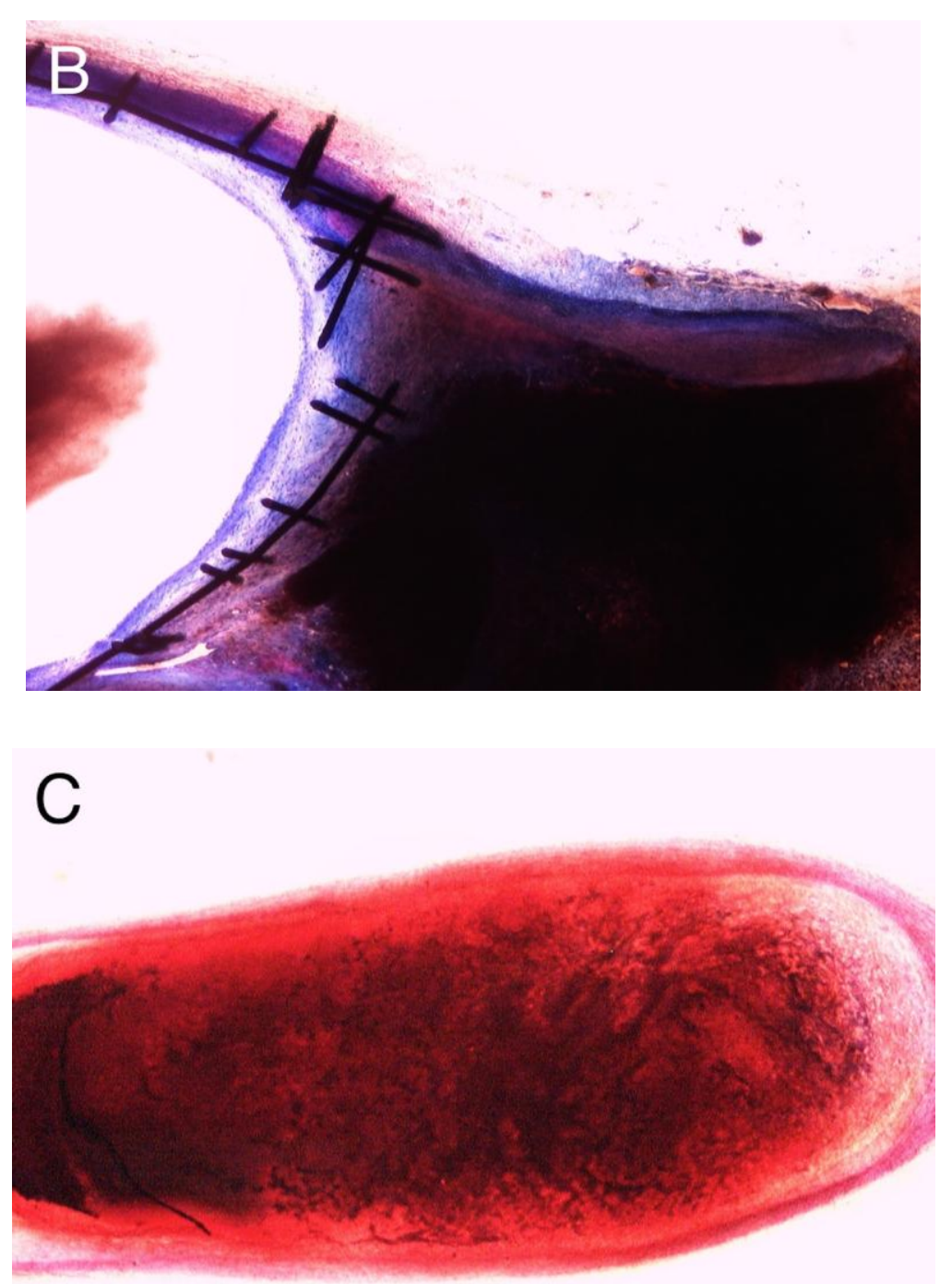

Figure 3 Histological micrographs showing the lines of Zahn: (A) rabbit A; (B) rabbit B; (C) rabbit $\mathrm{C}$

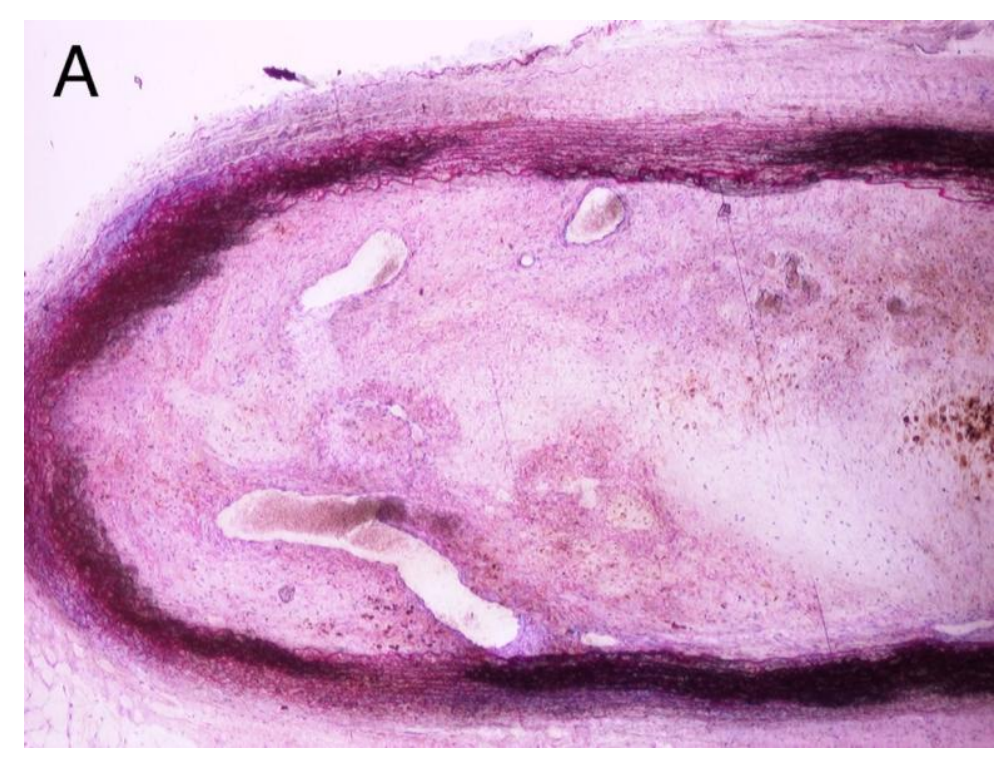



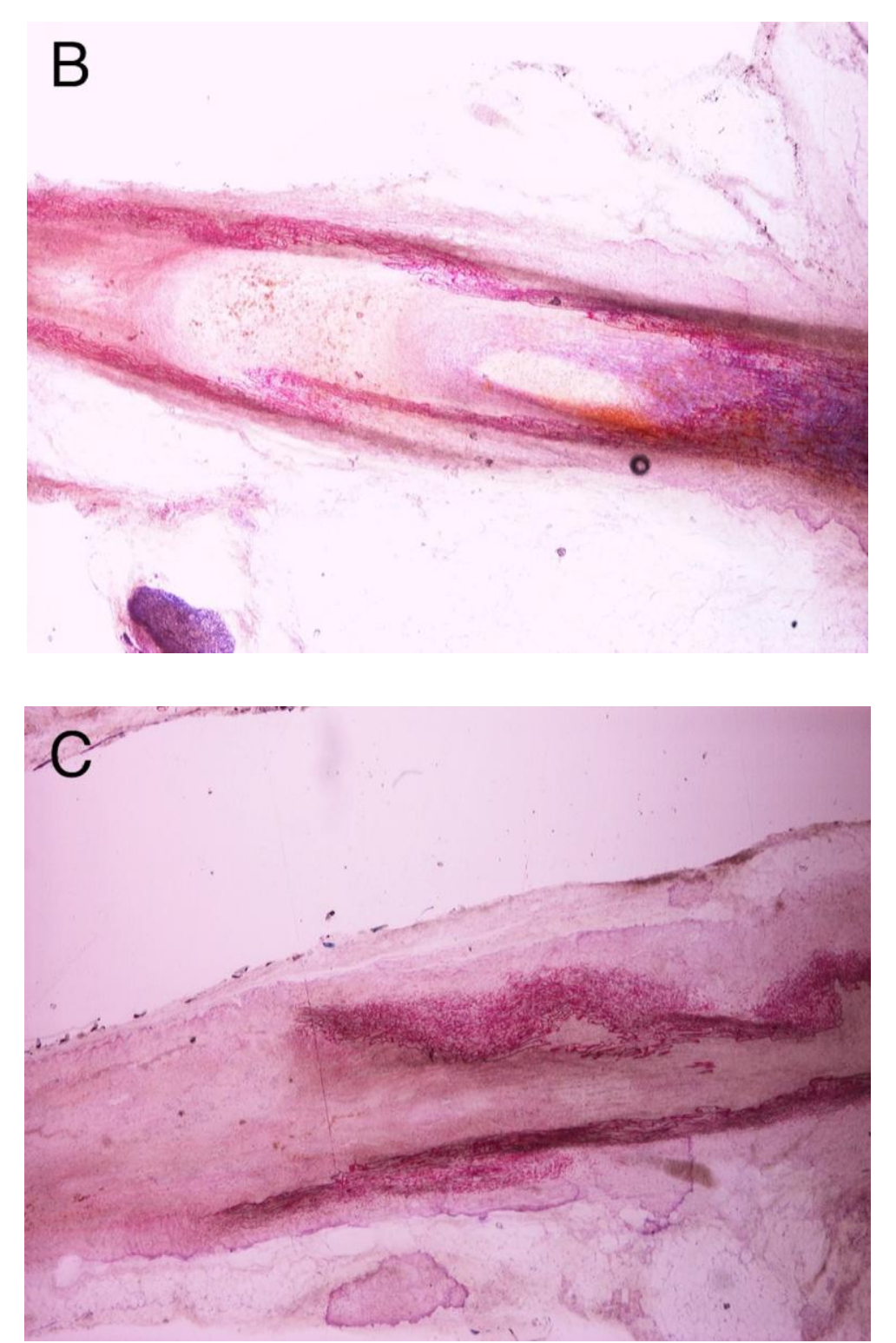

Figure 4 Histological micrographs showing characteristics of a vascularized fibroconnective tissue: (A) rabbit D; (B) rabbit E; (C) rabbit F - remodeled thrombus

\subsection{EDAX Analysis}

EDAX analysis of the specimen from rabbit $\mathrm{C}$ revealed that in all areas investigated in the tissues surrounding the device struts, there was an absence of nickel and titanium (Figure 5A). An EDAX analysis on the uncovered struts, which was carried out as a control, showed the presence of nickel and titanium (Figure 5B). There were some areas of struts showing signs of superficial defects. However, it was unclear whether the loss of material occurred prior to device placement, during the implantation or postimplantation period. Nevertheless, there was an absence of nickel and titanium in the surrounding tissues. 

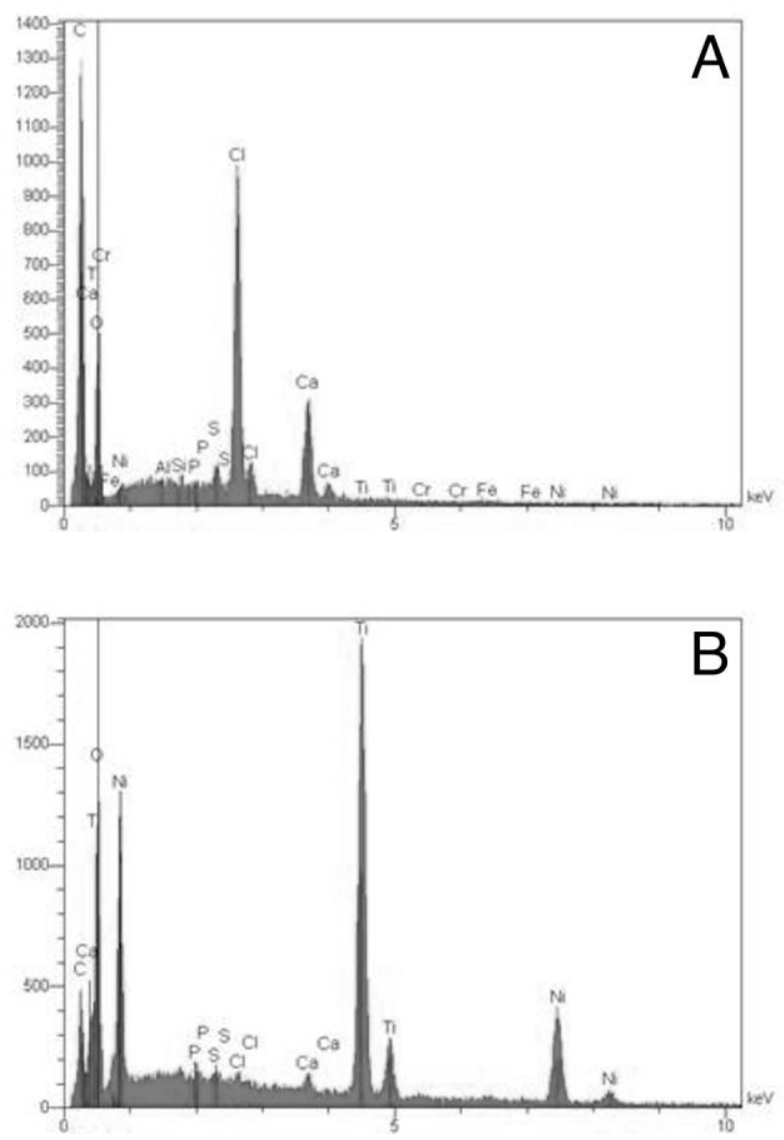

Figure 5 EDAX analysis: (A) rabbit $\mathrm{C}$ - absence of nickel and titanium; (B) rabbit $\mathrm{C}$ (control)

\section{DISCUSSION}

This study involved the assessment of the intracranial MFM device in rabbit animal models, specifically an elastase induced saccular aneurysm. Significantly, all specimens showed red thrombus deposits, ranging from a moderate to marked grade of deposition in the subclavian segment. Histopathological analysis further demonstrated obturation of the aneurysmal area by a mixed thrombus showing the lines of Zahn. These results attested to a low blood flow, and thrombus remodeling. This phenomenon of intra arterial remodeling is the basis of the MFM design. As blood flows through the device mesh, it is modulated and slowed. This slowing directly causes organized thrombus formation and aneurysm stabilization. The reduction in blood flow velocity also reduces shear stress on the aneurysm sac wall, thereby reducing the risk of rupture. In the case of saccular aneurysms, with no collateral arteries, obturation of the aneurysm from blood flow by the MFM is desired, effectively removing the problematic aneurysm from circulation.

\subsection{Animal Models}

Animal models are commonly used as a first step method of assessment for new endovascular technologies (Ionita et al. 2009; Kallmes et al 2009). Previous animal studies have examined the use of 'flow diverters' for intracranial aneurysms. These devices are similar in concept to the MFM. A previous study by Ionita et al. examined an AVS, which contained a low porosity patch. Twenty-four elastase rabbit model saccular aneurysms were created, 13 of which were treated with the AVS. 
Following a 4 week study period, angiographic results showed that the AVStreated aneurysms exhibited very weak or no aneurysm flow immediately after treatment and no flow in all aneurysms at 4 weeks. SEM showed that all AVS aneurysms were occluded, while histology results demonstrated advanced thrombus organization. A similar study by Kallmes et al. studied a braided endoluminal device for saccular aneurysm occlusion. The device was implanted across the necks of 18 saccular induced aneurysms in rabbit models. The rabbits were subsequently followed for 1, 3 and 6 months. Seventeen cases of complete occlusion were recorded. No incident of branch occlusion or distal emboli in vessels downstream of the parent artery was observed. Parent artery neointimal hyperplasia was also minimal.

Study methods from Ionita et al. and Kallmes et al. compare to this study. The aneurysms in this study were also elastase induced. Histology and SEM analysis were conducted previously while EDAX was used in this study to assess the tissue surrounding the device struts for metal content. These evaluation methods are valuable long-standing tools in establishing biocompatibility of vascular endoprosthesis. Histology was used to examine the intra-arterial biocompatibility of the devices.

These animal studies from the literature using this model makes this work a coherent addition, and appropriate for translational research.

\subsection{Clinical Outcomes}

An estimated 6 million people in the United States have an unruptured brain aneurysm, or 1 in 50 people (Understanding: Brain Aneurysm Statistics and Facts). Each treatment case is unique in terms of aneurysm location, its shape and its size. The most common types of treatment are aneurysm clipping or coil embolization (Dinç et al. 2013; Brown et al. 2014). A recent epidemiologic review study by Kelly (2014) indicated that the overall prevalence of intracranial aneurysms to be approximately $3 \%$, with higher rates seen in familial aneurysm syndromes and in certain medical conditions. This study also highlighted the increasing utilization of endovascular strategies over time. Increased aneurysm diameter, certain locations, and other anatomical considerations may be associated with higher risks of aneurysm rupture. Previously literature concerning intracranial flow diverters has yielded positive results, with high aneurysm occlusion reates (Brinjikji et al. 2013; Rajpal et al. 2013).

To date, the MFM has already been successfully translated to human research (Henry et al. 2008; Polydorou et al. 2010; Chocron et al. 2011; Ferrero et al. 2011; Meyer et al. 2011; Sultan et al. 2012;), however there is currently no published literature on the intracranial MFM. A clinical trial to evaluate treatment of intracranial aneurysms using the MFM was conducted between September 2010 and June 2013. It was subsequently presented at The World Federation of Interventional and Therapeutic Neuroradiology in 2013 by Gál et al. (2013). Seventy-one patients with 89 brain aneurysms were treated with the intracranial MFM. Nine of the aneurysms were fusiform and 29 aneurysms were bifurcational, with branches extending from the sac. Eleven of them were large, 3 giant, the remaining small. Six to 12 months following treatment in 26 patients, 22 aneurysms in 20 patients were totally occluded, 3 partially occluded, and 3 aneurysms were 
unchanged. The trial concluded that the cranial device is a promising new device. It has the potential to promote intrasaccular thrombosis and successive shrinkage of all kinds of aneurysms, including bifurcational aneurysms, at all intracranial locations. In terms of aneurysm occlusion, the results of this study compare favorably with flow diverter data published previously (Brinjikji et al. 2013; Rajpal et al. 2013). The MFM is current being used successfully to treat peripheral, visceral and aortic aneurysms, (Henry et al. 2008; Polydorou et al. 2010; Chocron et al. 2011; Ferrero et al. 2011; Meyer et al. 2011; Sultan et al. 2012;), but these initial results show that the intracranial MFM offers a new minimally invasive treatment for intracranial aneurysms, alongside currently available flow diverters.

\section{CONCLUSIONS}

In this evaluation of the intracranial MFM, obturation of the saccular aneurysmal area by a mixed thrombus, showing the lines of Zahn was recorded. This in turn was associated with signs of thrombus remodeling. These results demonstrate the basic mechanism of the MFM, its ability to modulate flow from turbulent to laminar, thereby encouraging organized thrombus formation. Studies using a larger cohort are necessary to further assess hemocompatibility, local and systemic safety and effectiveness.

\subsection{Limitations}

This study was limited by the use of young and otherwise healthy rabbit test animals. The results showed the devices were mostly positioned and deployed without any difficulty in the test animal arteries. However due to the above limitation, this does not compare to a surgical situation. Furthermore, animal studies are limited by design. They often automatically control many variables, which would otherwise occur in a normal human surgical situation (Hackam et al. 2006). Animal studies therefore do not include naturally occurring variation (Williams et al. 2004). 


\section{REFERENCES}

Bouzeghrane F, Naggara O, Kallmes DF, Berenstein A, Raymond J, International Consortium of Neuroendovascular Centres. "In vivo experimental intracranial aneurysm models: a systematic review." AJNR Am J Neuroradiol 31, no. 3 (2010): 418-423. doi: 10.3174/ajnr.A1853.

Brinjikji W, Murad MH, Lanzino G Cloft HJ, Kallmes DF. "Endovascular treatment of intracranial aneurysms with flow diverters: a meta-analysis." Stroke 44, no. 2 (2013): 442-447. doi: 10.1161/STROKEAHA.

Brown RD, Broderick JP. "Unruptured intracranial aneurysms: epidemiology, natural history, management options, and familial screening." Lancet Neurol 13, no. 4 (2014): 393-

404. doi: 10.1016/S14744422(14)70015-8.

Byrne JV, Szikora I. "Flow Diverters in the Management of Intracranial Aneurysms: A Review." EJMINT. (2012): 1225000057. Accessed February 11, $2014 . \quad$ url: http://www.ejmint.org/originalarticle/1225000057.

Chocron S, Vaislic C, Kaili D, Bonneville JF. "Multilayer stents in the treatment of thoraco- abdominal residual type B dissection." Interact Cardiovasc Thorac Surg 12, no. 6 (2011): 1057-1059. doi: 10.1510/icvts.2010.257394.

Dinç H, Öztürk MH, Sari A, Çakir E, Gazioğlu G, Kuzeyli K. "Coil embolization in 481 ruptured intracranial aneurysms: angiographic and clinical results." Diagn Interv Radiol 19, no. 2 (2013): 165-172. doi: 10.4261/13053825.DIR.6197-12.1.

Donath K, Brunner G. "A method for the study of nondecalcified bone and teeth with attached soft tissues." J Oral Pathol Med 11, no. 4 (1982): 318-326. DOI: $10.1111 / \mathrm{j} .1600-$ 0714.1982.tb00172.x.

Ferrero E, Ferri M, Viazzo A, Robaldo A, Carbonatto P, Pecchio A, Chiecchio A, Nessi F. "Visceral Artery Aneurysms, an Experience on 32 Cases in a Single Center: Treatment From Surgery to Multilayer Stent." Ann Vasc Surg 25, no. 7 (2011): 923- 35. doi: 10.1016/j.avsg.2011.04.006.

Gál G, Rudnicka S. "Mid term experiences with a new flow diverter, the Cardiatis Intracranial Multilayer System", Presentation at the $12^{\text {th }}$ congress of the World Federation of Interventional and Therapeutic Neuroradiology, Buenos Aires. November 9-13 2013.

Hackam DG, Redelmeier DA. "Translation of Research Evidence From Animals to Humans." J Am Med Assoc 296, no. 14 (2006): 1731-1732. doi:10.1001/jama.296.14.1731.

Henry M, Polydorou A, Frid N, Gruffaz P, Cavet A, Henry I, Hugel M, Rüfenacht DA, Augsburger L, De Beule M, Verdonck P, Bonneau M, Kang C, Ouared R, Chopard B. "Treatment of Renal Artery Aneurysm With the Multilayer Stent." J Endovasc Ther 15, no. 2 (2008): 231-236. doi: 10.1583/072222.1 .

Ionita $\mathrm{CN}$, Paciorek AM, Dohatcu A, Hoffmann KR, Bednarek DR, Kolega J, Levy EI, Hopkins LN, Rudin S, Mocco JD. "The Asymmetric Vascular Stent: Efficacy in a rabbit aneurysm model." Stroke 40, no. 3 (2009): 959-965. doi: 10.1161/STROKEAHA.108.524124.

Kallmes DF, Ding YH, Dai D, Kadirvel R, Lewis DA, Cloft HJ. "A Second-Generation, Endoluminal, FlowDisrupting Device for Treatment of Saccular Aneurysms." Am J Neuroradiol 
30, no. 6 (2009): 1153-1158. doi: 10.3174/ajnr.A1530.

Kelly AG. "Unruptured Intracranial Aneurysms: Screening and Management." Continuum (Minneap Minn) 20, no. 2 Cerebrovascular Disease (2014): 387-398. doi:

10.1212/01.CON.0000446108.12915.65.

Lubicz B, Collignon L, Raphaeli G, Pruvo JP, Bruneau M, De Witte O, Leclerc $X$. "Flow- Diverter Stent for the Endovascular Treatment of Intracranial Aneurysms - A Prospective Study in 29 Patients With 34 Aneurysms." Stroke 41, no. 10 (2010):

doi:

10.1161/STROKEAHA.110.589911.

Meyer C, Verrel F, Weyer G, Wilhelm K. "Endovascular Management of Complex Renal Artery Aneurysms Using the Multilayer Stent." Cardiovasc Intervent Radiol 34, no. 3 (2011): 637-641. doi: 10.1007/s00270-010-0047-0.

Piotin M, Blanc R, Spelle L, Mounayer C, Piantino R, Schmidt PJ, Moret J. "Stent-assisted coiling of intracranial aneurysms: clinical and angiographic results in 216 consecutive aneurysms." Stroke 41, no. 1 (2010): 110115.

10.1161/STROKEAHA.109.558114.

Polydorou A, Henry M, Bellenis I, Kiskinis D, Bolos K, Athanasiadou K, Portinos A, Dedeilias P, Kokotsakis I, Anthopoulos P, Chondros G, Testempasi E, Farsaris D, Kratimenos T, Tsiakouri C, Papapavlou P, Rammos S, Perdikides T, Polydorou A, Polydorou V, Stavrou G, Megalooikonomos P, Moutiris J, Fotis T. "Endovascular treatment of arterial aneurysms with side branches - a simple method. Myth or reality?" Hospital Chronicles 5, no. 2 (2010): 88-94. url: http://www.hospitalchronicles.gr/index.ph $\mathrm{p} / \mathrm{hchr} /$ article/view/214
Rajpal G, Kadzioloka K, Estrade L, Pierot L. "Flow diverter neuroendovascular stents- Reconstructive endovascular treatment of intracranial aneurysms-Single centre experience" Indian J Neurosurg 2, no. 2 (2013): 182188. url: http://www.ijns.in

Sultan S, Hynes N. "Disruptive Endovascular Technology with Multilayer Flow Modulator Stents as a Therapeutic Option in the Management of Thoracoabdominal Aortic Aneurysms. Early Results From the Global Independent MFM Registry." Italian J Vasc Endovasc Surg 19, no. 4 (2012): 215228.

url: http://www.minervamedica.it/en/journals/v ascular-endovascular-

surgery/article.php?cod=R46Y2012N04A0 215

"Understanding: Brain Aneurysm Statistics and Facts." Brain Aneurysm Foundation. Last modified, 2015. http://www.bafound.org/Statistics_and_Fa cts.

Williams SM, Haines JL, Moore JH. "The use of animal models in the study of complex disease: all else is never equal or why do so many human studies fail to replicate animal findings?" Bioessays 26, no. 2 (2004): 170-179. doi: 10.1002/bies. 10401

Wong GK, Kwan MC, Ng RY, Yu $\mathrm{SC}$, Poon WS. "Flow diverters for treatment of intracranial aneurysms: current status and ongoing clinical trials." J Clin Neurosci 18, no. 6 (2011): 737-740. doi: 10.1016/j.jocn.2010.10.011. 